\title{
Intensity of medical care in internal medicine: impact on outcomes from a trend analysis over six years
}

\author{
Walter Spagnolli, ${ }^{1}$ Dimitri Peterlana, ${ }^{1}$ Stefania Dorigoni, ${ }^{1}$ Marta Rigoni, ${ }^{2}$ Emanuele Torri, ${ }^{3}$ Giandomenico Nollo ${ }^{2,4}$ \\ ${ }^{1}$ Internal Medicine Department, Santa Chiara Hospital, Trento Local Healthcare Trust, Trento; ${ }^{2}$ Healthcare Research and \\ Innovation Program-Heath Technology Assessment Unit, Fondazione Bruno Kessler, Trento; ${ }^{3}$ Department of Health and Social \\ Policies, Autonomous Province of Trento; ${ }^{4}$ BioTech Lab, Department of Industrial Engineering, University of Trento, Italy
}

\begin{abstract}
In large acute medical wards treating heterogenous and complex patients, intensity of patient care should be graduated according to clinical severity. We conducted a retrospective observational study on all unselected admissions (8838) to the internal medicine ward of the Santa Chiara Hospital of Trento from 2012 to 2017. During 2012 and 2013, a standard organizational model (SMC) was in place, while an organizational model for intensity of medical care (IMC) was introduced in 2014. In SMC, patient admission was performed according to bed availability only. In IMC, patients were allocated to three different ward settings (high, medium and post-acute care) based on the stratification of clinical instability. The National Early Warning Score (NEWS) was used for the stratification, together with the clinical judgment. The implementation of the IMC model led to a decrease of mortality and urgent transfers for clinical deterioration to Intensive Care Unit and to an increase of admissions from Intensive Care Unit and from regional spoke hospitals. Redesigning delivery processes based on IMC can play a pivotal role in improving patient outcomes and bed management.
\end{abstract}

Correspondence: Marta Rigoni, Healthcare Research and Innovation Program-Heath Technology Assessment Unit, FBK, via Sommarive 18, 38123 Trento, Italy.

Tel.: +39.0461.314664. E-mail: mrigoni@fbk.eu

Key words: Intensity of medical care; patient risk stratification; early warning score; organizational change; bed management.

Contributions: WS, substantial contributions to the conception of the work; GN, ET, MR, substantial contributions to the design of the work; WS, DP, SD, data acquisition; MR, data analysis; MR, ET, data interpretation; GN, MR, ET, drafting the work and revising it critically for important intellectual content; GN, MR, ET, WS, DP, SD, final approval of the version to be published.

Conflict of interests: the authors declare no potential conflict of interests.

Conference presentation: in 2018, this work was partially presented at the following National Conferences: Federation of Associations of Hospital Doctors on Internal Medicine (FADOI), Italian Society of Health Technology Assessment (SIHTA), Italian Society of Hygiene, Preventive Medicine and Public Health (SITI).

Received for publication: 30 January 2019.

Revision received: 1 April 2019.

Accepted for publication: 2 April 2019.

This work is licensed under a Creative Commons Attribution NonCommercial 4.0 License (CC BY-NC 4.0).

CC Copyright W. Spagnolli et al., 2019

Licensee PAGEPress, Italy

Italian Journal of Medicine 2019; 13:95-102

doi:10.4081/itjm.2019.1140

\section{Introduction}

All over the western world, internal medicine wards are experiencing an increase in workload and number of admissions of heterogeneous, complex, frail, and vulnerable patients for which, in many cases, there is no single best pathway of diagnosis and management. In internal medicine wards such cases constitute the majority of urgent hospital admissions, with often undefined diagnosis and different levels of clinical stability, varying from stable to unstable or critical conditions. ${ }^{1,2}$ Therefore, it is crucial to go beyond traditional organizational models of general medical wards shaped as undifferentiated settings of care, relying on clinical speciality and providing a diluted $a v-$ erage standard of care for all patients. This may lead to unmet or mismatched needs, especially for critically ill patients, vulnerable to clinical deterioration, resulting in an increased risk of adverse events and suboptimal quality of care. ${ }^{3}$ Identification of patients at risk of clinical deterioration during hospital stay is critical to deliver safe and effective care in acute medical care.

Understanding how to better organize health care for general medical patients is an international priority. ${ }^{4}$ Indeed, more than any other health care setting, general medical wards generate the errors that lead to preventable deaths. ${ }^{5}$ Intensive care admissions may be preventable in $21 \%$ of cases and suboptimal care may be responsible for up to one-third of hospital deaths. ${ }^{6,7}$

Medical wards lay at the heart of inpatient medicine but, perhaps more than any other delivery setting of acute hospitals, are facing the daunting challenge 
of conjugating optimal levels of safety, effectiveness and patient-centeredness of care with efficiency and accountability in the use of the limited resources available. Models proposed to solve such dilemma are centered around the paradigm of organizing care delivery and management of patient flow providing the required intensity of medical care. ${ }^{8,9}$

Such approach applied to medical wards relies on the classification of patient acuity performed using tools for stratification of severity of patient conditions and the provision of levels of progressive care, stepping up and down patients, according to clinical stability and complexity of patient needs.

Patients should be clustered by severity, in order to identify their clinical risk, at the time of ward admittance using validated prognostic scores and allocated to areas dedicated to patients with homogeneous needs of care and level of acuity. ${ }^{10}$ Among the different scores proposed for early risk stratification, the National Early Warning Score (NEWS) has been recognized as an effective tool for routine application in a large internal medicine ward ${ }^{11}$ and recently recommended by the Royal College of Physicians in all hospital and non hospital settings. ${ }^{12}$

According to the paradigm of intensity of medicalcare (IMC), patients can be managed and progressively moved (stepped up and down), based on their clinical condition, among ward areas with different, but strongly integrated, levels of care. Addressing these changes to achieve real improvement requires technological and staffing arrangements, collaboration among medical and nursing staff members, effective clinical protocols and regular feedback on achievements. To our knowledge, despite the growing interest of clinicians and healthcare organizations on the issue of application of IMC in internal medicine, there are still limited published data on sustainability of improvement and changes on the impact on outcomes variable in the real context of general medicine wards.

In a recently published study, ${ }^{9}$ we showed the effectiveness of IMC approach over a short period. That study aimed to assess outcomes stricltly before and after the implementation of such innovative model, but it neither investigated nor provided any information about the robustness and durability of the benefits over a long period. The complexity of the intervention made further research and efforts mandatory, and this led to an increase of timeframe and breadth of the analysis.

The purpose of our study was to show how the reorganization of a large Internal Medicine ward, with the introduction of an IMC model supported by patient clinical risk stratification and performed according to the NEW score, can affect clinical and organizational outcomes over a long period of time (six years) and on a large patient population. We discuss reasons why we believe blending appropriate assessment and grad- uated response to complexity of patients, may provide a pivotal option to tackle major issues challenging care delivery in internal medicine.

\section{Materials and Methods}

\section{Design and setting}

This study is a retrospective observational study performed in the Internal Medicine (IM) ward of the Santa Chiara Hospital of Trento. Santa Chiara Hospital is the main facility of the public hospital system managed by the Healthcare Trust of the Autonomous Province of Trento. The hospital plays a pivotal role (hub) in the acute hospital network of the region, which is organized according to a hub and spoke model. The Autonomous Province of Trento is an alpine region in north-eastern Italy, with over 530,000 inhabitants and a surface area of $6212 \mathrm{~km}^{2}$.

\section{Standard of medical care}

Until the end of 2013, the IM ward consisted of three clinical sections, each with its own organization, for 58 beds. The organizational model was traditional with bed assignment reflecting the principle of the first available bed only. Telemetry-monitored beds, excluding electrocardiography, were unavailable. One joint (physician and nurse) ward round was performed daily with clinical revaluations if necessary; sporadically it was possible to perform bedside non-invasive mechanical ventilation.

\section{Intensity of medical care}

From 2014 to the end of 2017, the IM Unit has operated the ward with 56 beds differentiated into three integrated ward areas managed by intensity of medical care: i) High Care Area providing high-intensity medical care or intermediate care (intermediate care area, ICA) with 8 beds equipped with centralised multi-parameters monitoring technology (electrocardiography, pulse oximetry, non-invasive blood pressure measurement); possibility to perform non-invasive ventilation; twice-a-day joint (physician and nurse) ward rounds and clinical revaluations if necessary; ii) Medium Care Area, providing a medium-intensity medical care (medium care area, MCA) and consisting of 33 beds with the capability of telemetric monitoring (electrocardiography), one daily joint (physician and nurse) ward round and clinical revaluations if necessary; iii) Post-Acute Area, providing low-intensity medical care (post-acute care area, PACA) and consisting of 13 beds; one daily joint (physician and nurse) ward round and clinical revaluations if necessary. In this area, urgent admissions were not planned, but only transfers of patients in post-acute phase stepping down from 
higher levels of care (ICA and MCA) needing clinical/functional rehabilitation and/or with discharge problems.

Under this organizational model, patient bed assignment to different areas of the ward followed an assessment on ward admission in a common area and allocation to ICA (high-intensity area) or MCA (medium-intensity area) depending on the degree of clinical instability and the related risk of clinical deterioration. Since July 2013, all patients were triaged on admission by nursing staff trained to collect and understand the six physiological parameters routinely recorded for NEWS calculation. The NEWS is based on a simple measurement system in which a score is allocated to physiological parameters collected by nursing staff at bedside, without any additional workload beside routine clinical monitoring activity. On admission, nurses collected each of the clinical findings forming the basis of the scoring system: i) respiratory rate; ii) oxygen saturation; iii) temperature; iv) systolic blood pressure; v) pulse rate; and vi) level of consciousness, with assessment of level of response on AVPU scale (alert, voice, pain, and unresponsive). As recommended by the Royal College of Physicians, ${ }^{13}$ patients were grouped into the following three trigger levels/risk categories: low score (NEW score, 0-4); medium score (NEW score, 5-6); and high score (NEW score, $\geq 7$ ).

Physicians provided appropriate strategy for response and setting of care relying on NEWS and clinical judgment.

\section{Participants}

All the adult patients consecutively admitted to the IM Unit of the Santa Chiara hospital in Trento, from $1^{\text {st }}$ January 2012 to $31^{\text {th }}$ December 2017 were included in the study. From $1^{\text {st }}$ January 2012 to $31^{\text {th }}$ December 2013 , the organization of the ward was based on traditional standard medical care service (SMC). IMC model started to be implemented since $1^{\text {st }}$ January 2014, so from $1^{\text {st }}$ January 2014 to $31^{\text {th }}$ December 2017, ward organization was based on IMC. Patients were admitted to the IM ward from the Emergency Room, from other acute care hospitals and from the Intensive Care Unit (ICU) in a post-critical phase.

\section{Statistical analyses and outcomes}

Descriptive statistics were expressed in numbers, percentages, mean and standard deviation, and median and interquartile range, when appropriate. In order to analyse the key outcomes over the six-year period, we performed a trend analysis for proportions based on chi-square statistic. The organizational change was analysed through the following patient outcomes: i) early in-hospital mortality (within $72 \mathrm{~h}$ of admission); ii) total in-hospital mortality; iii) urgent (unanticipated and unplanned) transfers for clinical deterioration to a higher level of care (intensive care): ICU, Intensive Coronary Unit, Intensive Respiratory Unit; iv) combined outcome one: early in-hospital mortality and urgent transfers for clinical deterioration; v) combined outcome two: total mortality and urgent transfers for clinical deterioration.

In addition, we assessed the following performances related to management of bed capacity, patient flow and reliability of scoring system: i) proportion of stepdown patients admitted to IM ward transferred from a higher level of care; ii) proportion of unstable medical patients transferred to IM ward from spoke hospitals; iii) bed occupancy; iv) nurse-to-patient ratio.

Data were collected in an aggregate way from the Hospital Informative System. During the analysis, we did not collect and manage sensitive data as well as encodings able to directly and univocally identify the individual patient. In accordance with the data management approach of the local healthcare trust, patient informed consent was not necessary. According to the rules of the Healthcare Trust of the Autonomous Province of Trento, no ethical committee approval was needed.

A P-value less than 0.05 indicated statistical significance. All analyses were performed with STATA statistical software, version 13.0 (StataCorp, College Station, TX, USA).

\section{Results}

We analysed 1453 and 1393 consecutive admissions during SMC period (2012 and 2013) and 1437, 1622,1498 , and 1435 consecutive admissions during IMC period $(2014,2015,2016$ and 2017) with a total population of 8838 .

The characteristics of the study population (patients treated under SMC and IMC) are summarised in Table 1. We did not find significant differences in demographic data and average weight of diagnosis related groups (DRGs) over the six years. Most frequent causes of admission were cardiac failure, acute respiratory illness and sepsis. Over 95\% were urgent medical admissions.

\section{Total in-hospital mortality}

Total in-hospital mortality varied from $8.7 \%$ in 2012 to $7.0 \%$ in 2017 , showing a statistical dropping trend over the six-year period, $\mathrm{P}=0.019$ (Table 2, Figure 1).

\section{Early in-hospital mortality (within $72 \mathrm{~h}$ of admission)}

We found a significant decreasing trend of early death over the six years: from $3.5 \%$ in 2012 to $2.2 \%$ in 2017, $\mathrm{P}=0.002$ (Table 2, Figure 1). 


\section{Urgent transfers to intensive care}

Urgent and unplanned transfers to intensive care changed from $4.2 \%$ in 2012 to $1.6 \%$ in 2017 , showing a meaningful decreasing trend over the six-year study, $\mathrm{P}<0.001$ (Table 2, Figure 1).

\section{Combined outcome one} (early in-hospital mortality and urgent transfers)

We found a significant reduction $(\mathrm{P}<0.001)$ in the combined outcome over the analysed years: from $7.7 \%$ to $3.8 \%$ for 2012 and 2017 respectively (Table 2).

\section{Combined outcome two (total in-hospital mortality and urgent transfers to intensive care)}

We found a significant reduction $(\mathrm{P}<0.001)$ in the combined outcome over the analysed years: from $12.2 \%$ to $8.6 \%$ for 2012 and 2017 respectively (Table 2).

\section{Stepdown admissions from a higher level of care}

The proportion of stepdown admissions over the six-year period showed a significant trend increase from $1.9 \%$ in 2012 to $5.2 \%$ in $2017, \mathrm{P}<0.001$ (Table 2, Figure 2).

Table 1. Characteristics of the study population.

\begin{tabular}{|c|c|c|c|c|c|c|}
\hline Characteristic & $\begin{array}{c}\text { Year } 2012 \\
\text { SMC, n }(\%)\end{array}$ & $\begin{array}{c}\text { Year } 2013 \\
\text { SMC, n }(\%)\end{array}$ & $\begin{array}{c}\text { Year } 2014 \\
\text { IMC, } \mathbf{n}(\%)\end{array}$ & $\begin{array}{c}\text { Year } 2015 \\
\text { IMC, n (\%) }\end{array}$ & $\begin{array}{c}\text { Year } 2016 \\
\text { IMC, } \mathbf{n}(\%)\end{array}$ & $\begin{array}{c}\text { Year } 2017 \\
\text { IMC, } \mathbf{n}(\%)\end{array}$ \\
\hline Male & $738(51)$ & $701(50)$ & $720(50)$ & $890(55)$ & $827(55)$ & $795(55)$ \\
\hline Female & $715(49)$ & $692(50)$ & $717(50)$ & $732(45)$ & $671(45)$ & $640(45)$ \\
\hline Total median age [IQR] years & $72[62-82]$ & $71[62-81]$ & $69[61-80]$ & $70[62-81]$ & $70[62-82]$ & $70[61-81]$ \\
\hline $\begin{array}{l}\text { Average weight of } \\
D R G s \pm \text { standard deviation }\end{array}$ & $1.16 \pm 0.77$ & $1.17 \pm 0.81$ & $1.15 \pm 0.82$ & $1.12 \pm 0.79$ & $1.13 \pm 0.75$ & $1.19 \pm 0.77$ \\
\hline First DRG & $\begin{array}{l}\text { Cardiac failure } \\
\text { and shock } \\
150(10.3)\end{array}$ & $\begin{array}{c}\text { Pulmonary } \\
\text { edema and } \\
\text { respiratory failure } \\
177(12.7)\end{array}$ & $\begin{array}{l}\text { Pulmonary } \\
\text { edema and } \\
\text { respiratory failure } \\
204(14.2)\end{array}$ & $\begin{array}{c}\text { Pulmonary } \\
\text { edema and } \\
\text { respiratory failure } \\
212(13.1)\end{array}$ & $\begin{array}{c}\text { Pulmonary } \\
\text { edema and } \\
\text { respiratory failure } \\
185(12.3)\end{array}$ & $\begin{array}{l}\text { Cardiac failure } \\
\text { and shock } \\
156(10.9)\end{array}$ \\
\hline Second DRG & $\begin{array}{c}\text { Pulmonary } \\
\text { edema and } \\
\text { respiratory failure } \\
123(8.5)\end{array}$ & $\begin{array}{l}\text { Cardiac failure } \\
\text { and shock } \\
\text { e } 132(9.5)\end{array}$ & $\begin{array}{c}\text { Cardiac failure } \\
\text { and shock } \\
131(9.1)\end{array}$ & $\begin{array}{l}\text { Cardiac failure } \\
\text { and shock } \\
163(10.0)\end{array}$ & $\begin{array}{l}\text { Cardiac failure } \\
\text { and shock } \\
151(10.1)\end{array}$ & $\begin{array}{c}\text { Polmunary edema } \\
\text { and respiratory } \\
\text { failure } \\
155(10.8)\end{array}$ \\
\hline Third DRG & $\begin{array}{c}\text { Septicemia } \\
\text { without } \\
\text { mechanical } \\
\text { ventilation } \\
>17 \text { years } 75 \\
(5.2)\end{array}$ & $\begin{array}{c}\text { Septicemia } \\
\text { without } \\
\text { mechanical } \\
\text { ventilation } \\
>17 \text { years } 109 \\
(7.8)\end{array}$ & $\begin{array}{c}\text { Septicemia } \\
\text { without } \\
\text { mechanical } \\
\text { ventilation } \\
>17 \text { years } 90 \\
(6.3)\end{array}$ & $\begin{array}{c}\text { Septicemia } \\
\text { without } \\
\text { mechanical } \\
\text { ventilation } \\
>17 \text { years } 120 \\
(7.4)\end{array}$ & $\begin{array}{c}\text { Septicemia } \\
\text { without } \\
\text { mechanical } \\
\text { ventilation } \\
>17 \text { years } 108 \\
(7.2)\end{array}$ & $\begin{array}{c}\text { Septicemia } \\
\text { without } \\
\text { mechanical } \\
\text { ventilation } \\
>17 \text { years } 112 \\
(7.8)\end{array}$ \\
\hline Total & 1453 & 1393 & 1437 & 1622 & 1498 & 1435 \\
\hline
\end{tabular}

SMC, standard care; IMC, intensity of medical care; IQR, interquartile range; DRGs, diagnosis related groups; n.s., no statistically significant; cardiac failure and shock, DRG 127; pulmonary edema and respiratory failure, DRG 87 ; septicemia without mechanical ventilation $>17$ years, DRG 576.

Table 2. Studied outcomes and results during six-year period.

\begin{tabular}{|c|c|c|c|c|c|c|c|}
\hline Outcome & $\begin{array}{c}\text { Year } 2012 \\
\text { SMC, n (\%) }\end{array}$ & $\begin{array}{c}\text { Year } 2013 \\
\text { SMC, n }(\%)\end{array}$ & $\begin{array}{c}\text { Year } 2014 \\
\text { IMC, n (\%) }\end{array}$ & $\begin{array}{c}\text { Year } 2015 \\
\text { IMC, n (\%) }\end{array}$ & $\begin{array}{c}\text { Year } 2016 \\
\text { IMC, n (\%) }\end{array}$ & $\begin{array}{c}\text { Year } 2017 \\
\text { IMC, n (\%) }\end{array}$ & P-value* \\
\hline Early in-hospital mortality $(<72 \mathrm{~h})$ & $51(3.5 \%)$ & $47(3.4 \%)$ & $40(2.8 \%)$ & $31(1.9 \%)$ & $33(2.2 \%)$ & $32(2.2 \%)$ & 0.002 \\
\hline Total in-hospital mortality & $127(8.7 \%)$ & $100(7.2 \%)$ & $111(7.7 \%)$ & $109(6.7 \%)$ & $91(6.1 \%)$ & $100(7.0 \%)$ & 0.019 \\
\hline Urgent transfers to intensive care & $61(4.2 \%)$ & $70(5.0 \%)$ & $28(1.9 \%)$ & $28(1.7 \%)$ & $30(2.0 \%)$ & $23(1.6 \%)$ & $<0.001$ \\
\hline Combined outcome 1 & $112(7.7 \%)$ & $117(8.4 \%)$ & $68(4.7 \%)$ & $59(3.6 \%)$ & $63(4.2 \%)$ & $55(3.8 \%)$ & $<0.001$ \\
\hline Combined outcome 2 & $188(12.9 \%)$ & $170(12.2 \%)$ & $139(9.7 \%)$ & $137(8.4 \%)$ & $121(8.1 \%)$ & $123(8.6 \%)$ & $<0.001$ \\
\hline ICU stepdown & $28(1.9 \%)$ & $34(2.4 \%)$ & $62(4.3 \%)$ & $64(3.9 \%)$ & $61(4.1 \%)$ & $75(5.2 \%)$ & $<0.001$ \\
\hline Transfers from spoke hospitals & $58(4.0 \%)$ & $60(4.3 \%)$ & $108(7.5 \%)$ & $107(6.6 \%)$ & $126(8.4 \%)$ & $138(9.6 \%)$ & $<0.001$ \\
\hline Bed occupancy, \% & 85 & 87 & 93 & 99 & 96 & 94 & $<0.001$ \\
\hline Total & 1453 & 1393 & 1437 & 1622 & 1498 & 1435 & - \\
\hline
\end{tabular}

SMC, standard care; IMC, intensity of medical care; *P for trend of proportions. Combined outcome 1=urgent transfers for clinical deterioration and early in-hospital mortality; combined outcome $2=$ urgent transfer for deterioration and total in-hospital mortality. 


\section{Urgent transfers from spoke hospitals}

Urgent transfers from spoke hospitals over the study period changed from $4.0 \%$ in 2012 to $9.6 \%$ in 2017, showing a meaningful decreasing trend over the six-year study, $\mathrm{P}<0.001$ (Table 2, Figure 2).

\section{Organizational outcomes (bed occupancy and nurse-to-patient ratio)}

Bed occupancy changed over years, moving from $85 \%$ in 2012 to $91 \%$ in $2017, \mathrm{P}<0.001$ (Table 2, Figure 2 ) over the years. The nurse-to-patient ratio also changed from 1:9 in SMC model (2012, and 2013) to an average of 1:12 in IMC model (2014, 2015, 2016, and 2017). These ratios were 1:4, 1:12, and 1:14 in the ICA, MCA, and PACA respectively.
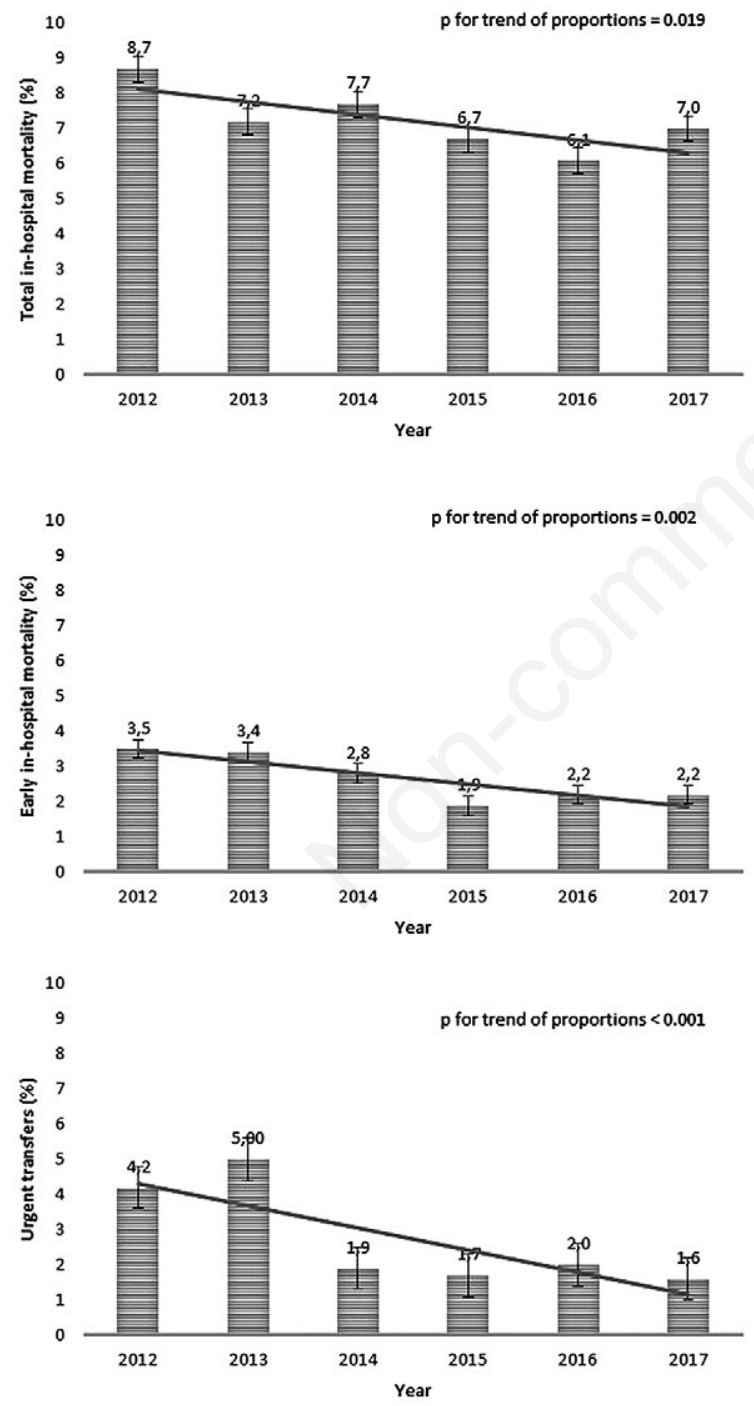

Figure 1. Clinical outcomes over six-year study period. The implementation of the intensity of medical care model, led to a continuous decrease of mortalities and urgent transfers due to clinical patient deterioration.

\section{Discussion}

This retrospective study of a multifaceted intervention in a large internal medicine ward demonstrated, over a six-year period, that the transition from SMC to IMC, was associated with a meaningful and sustained improvement in outcomes.

The organizational change determined by IMC was associated with a decreasing trend in the total inhospital mortality and in the early in-hospital mortality over the six-year period. Additionally, the number of unplanned transfers for clinical deterioration to intensive care units as well as the combination of unplanned transfers and mortality (combined outcome one and two) showed a continuous trend of reduction along the investigated period. Positive results were
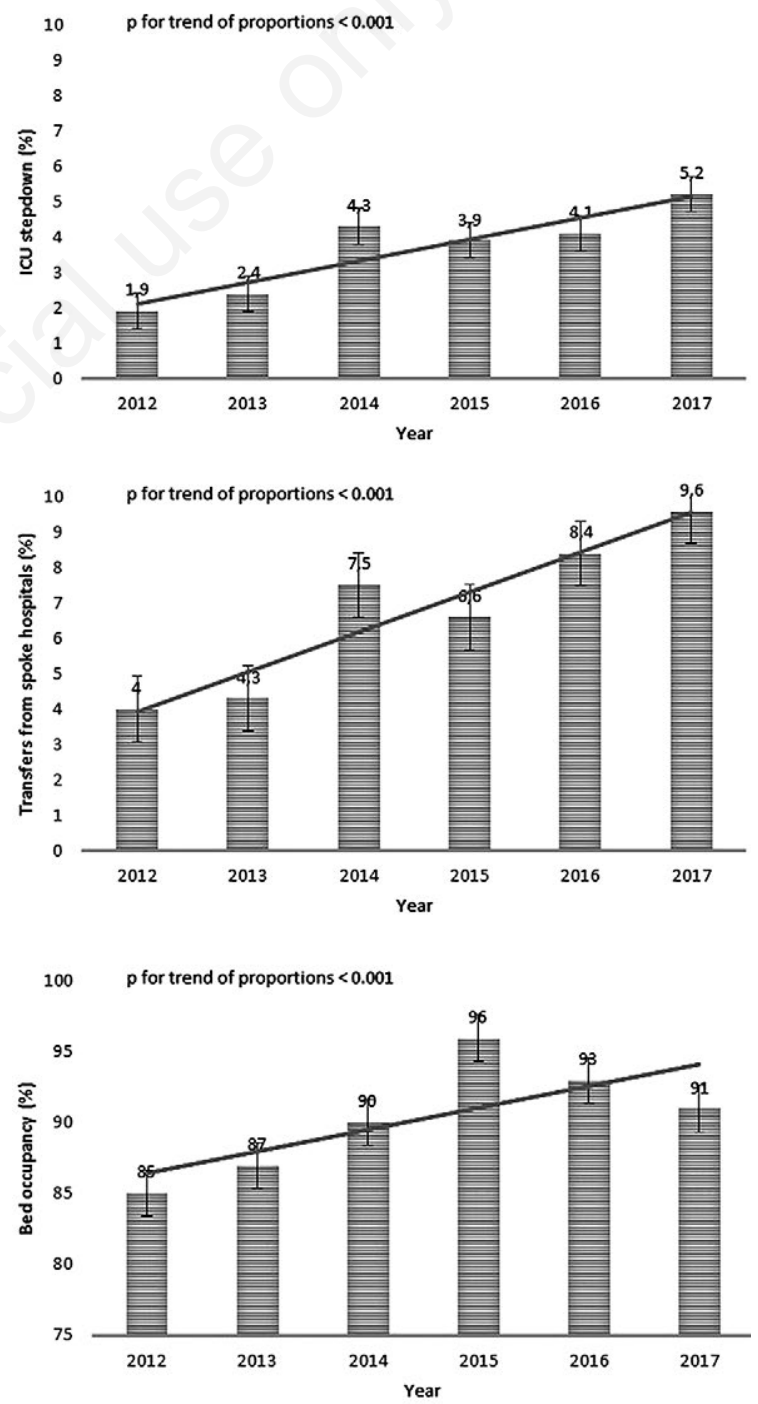

Figure 2. Organizational outcomes over six-year study period. The implementation of the intensity of medical care model, led to a continuous improvement of Intensive Care Unit (ICU) stepdown patients, patients from spoke hospitals, and bed occupancy. 
also the significant increase in patients admitted to the IM ward from intensive care (stepdown) and transferred for clinical instability from other acute medical wards of spoke hospitals of the regional hospital network. Finally, the introduction of the IMC model positively affected the management of the ward by increasing bed occupancy and nurse-to-patient ratio.

Therefore, our data show that systematic triage and stratification of patients based on risk of clinical instability using the NEWS and their allocation to the most appropriate setting of care throughout the course of hospitalization may improve clinical management and quality of care over the years.

In a previous work, ${ }^{9}$ we showed that the introduction of IMC determined a positive change in both clinical and organizative outocomes. Such study was an uncontrolled before-after study and we analysed data from two years: 2013 and 2015 for a total number of 3381 patients. In the study significant better results, according to a multivariate logistic regression model, were mainly associated with a reduction in urgent transfers to intensive care units. However, the study did not provide any information about the robustness of changes and outcome over a longer time frame underpinning the provision of the new model of care.

Widespread environmental pressures to improve standards of care in medical wards in Italy led to several recommendations, ${ }^{2,14-16}$ from professional bodies, scientific societies and healthcare institutions to implement plans and models that identify and mitigate barriers to safe and efficient patient flow providing appropriate intensity of medical care across the continuum of hospital care. This was recognized in Italy as key pattern of reorganization of acute hospitals, but without a full understanding of major ward interventions and transparent evaluation against a common set of outcomes over a long period in a real context of care.

Moreover, evidence of effective interventions to improve the quality and safety of care in medical wards is limited and it is unclear whether medical wards are truly resistant to strategies that have shown benefits elsewhere in the hospital. ${ }^{5}$ Furthermore, it has been reported that most studies showed little analysis of implementation and durability of the changes proposed. ${ }^{5}$

Some analogies with our experience of IMC may be found in experiences conducted in hospitals implementing intermediate care units, similar to our ICA. Such areas provided step-down beds in the hope that this would prevent a later ICU admission or step-up beds of patients from general wards or emergency departments. ${ }^{17}$ This can be a cost-effective solution to provide appropriate critical care outside intensive care units and to improve the flow of patients requiring more support than regular care, but not full intensive care. ${ }^{18,19}$

In our experience, the hospital setting for acute unstable patients was obtained through the implementa- tion, within the medical ward, of an ICA adequately equipped for monitoring and intervention, similarly to what has been reported in other studies on intermediate care beds. However, we believe that the sole implementation of ICA, which could resemble other studies on intermediate care/high dependency units, was not enough to explain the results achieved in the ward organization.

Setting up a new model of care requires systemwide changes in practice, that in our case were enabled by the commitment of clinical leadership, alignment and integration of clinical improvement efforts with organizational priorities. Other enablers included: medical and nursing staff competencies development, systematic collaboration among physicians and nurses and between internal medicine ward and intensive care units, adherence to evidence-based guidelines and protocols. It was also essential to establish an infrastructure for regular feedback on data, processes and performances. Our experience was implemented in a healthcare system pursuing a long-term comprehensive quality strategy. ${ }^{20}$

Baseline mortality data on in-hospital mortality at the IM ward of the Santa Chiara Hospital documented from 2010 to 2014 an increase in total mortality from $7.2 \%$ to $9.9 \%$. Among deaths, $38 \%$ to $42 \%$ of all cases occurred in the first $72 \mathrm{~h}$ of admission; indicating its clinical relevance as outcome measure. These results prompted immediate attention as the medical area manages a heterogeneous case mix and, as reported in medical literature, some deteriorating situations can go undetected.

In this regard, some issues concerning practice changes must be further highlighted. Since 2014, the implementation of the model took nearly two years of hard preparatory work in the field, undertaking challenging logistic, organizational and managerial steps with engagement of clinical staff and administrators. Regular feedback on achievements, shared with nursing and medical staff, solidified the practice changes and cleared up doubts on the value of the new organization of care.

The IMC model, based on graded care options requires a continuous collaborative organizational maintenance and encourages medical and nursing staff to take an active role either in the assessment of patient severity and in the identification of appropriate care settings.

That is critical for implementation of a new way of working in the routine care over the long term. The Quality Chasm is most likely to be crossed through a long slow journey rather than a single massive leap and it is important to recommend it. ${ }^{21}$ Therefore, it is important to support decision making and assess if changes translate into improvement over the longer term. 
We think that models of care improving risk stratification, patient flow and allowing a homogenous assessment of patient populations, should be advisable for organizations willing to embrace a new vision of value-based care.

The impact of change, variation of clinical practice and outcomes may be influenced by patient variables and related to complex organizational and context-related factors influencing systems of care. ${ }^{22,23}$ Influence of interventions on outcomes may require time and maturity, reflecting the developmental stage and sustainability of various quality improvement strategies and programs. ${ }^{24,25}$

Factors other than the new organizational model of care might explain changes in outcomes achieved in our study. As shown in results the patient population treated under SMC did not show any meaningful difference from the population treated under IMC, both considering demographic data and DRG.

Further, during the study period, the staff (nurses and physicians) yearly turnover rate (leavers in the year/total workforce) was below $10 \%$. We are not aware of any organizational factor that could have skewed our results. To reduce selection bias we included all the patients admitted during the two analyzed periods.

Other factors that could determine performances and outcomes include: staffing levels, nursing organization and competencies, as well as unit direction, organization and curriculum of physicians responsible for graded care options between intensive and conventional ward care. ${ }^{26}$

Our findings are indicative that during the investigated period under IMC, a lower proportion of urgent transfers to the intensive areas and it was increased number of stepdown patients admitted to MCA that the unit could manage. Considering the importance of availability of hospital beds in ICU for critical or postsurgical patients with a high severity of illness, ${ }^{19,27}$ this achievement per se means an optimized management of resources for the hospital system as a whole.

In addition, the new organization may be suggestive of more effective functioning of the provincial hospital network with the potential to admit a higher number of acute unstable medical patients transferred from the lower acuity settings of spoke hospitals to the larger and more comprehensive IM ward of Santa Chiara Hospital. A reservoir of beds for patients transfer from wards within the care network is crucial for the Hub \& Spoke model.

\section{Limitations}

Our study is a retrospective and single-center study. In particular, all clinical, demographical data, and outcomes were collected at the time of patient admission. Only the analysis was retrospective, thus re- ducing the risk of bias in measurement of outcomes. The single-center nature of the study that warrants a homogeneous patient management, on the other side could limit generalizability to other institutions.

We performed an observational study, which is probably the only option once beds have been reorganized and high-dependency beds set up, considering the great pressure to admit patients and ethical issues involved by the intervention implemented. To reduce selection bias, we included all patients admitted during the six-year period.

In our study, we used outcome measures, singularly or in combination, widely proposed in studies evaluating performances of EWS, impact of intermediate care models and more in general safety and quality of hospital care. ${ }^{28,29}$

We did not focus on and report any economic- and cost-related outcomes. Although, we used some quality outcomes that by definition are associated with a more appropriate use of hospital resources. Results show an improvement in bed management and patient flow, as well as a sustained occupancy rate higher than $90 \%$, which seems to provide, according to measured outcomes, a good balance between safety and care efficiency. Our data seem to indicate that IMC might allow adequate levels of care for patients even with occupation rates between $90 \%$ and $100 \%$, otherwise considered in traditional models of care a possible cause of higher risk of adverse events for patients. ${ }^{30}$

\section{Conclusions}

The IMC model, under a continuous collaborative organizational maintenance, led to a durable effect over a long period on clinical outcomes (including inhospital mortality and urgent transfers to a higher level of care) and ward management parameters of efficient management of patient flow and beds, thus indicating that well-settled graded care options can offer adequate level of safety and quality of care.

In the paper, we discussed the results of a long lasting implementation of a complex intervention requiring a combination of structural and process changes together with clinical leadership. We analyzed an organizational model that may be feasible and valuable targeting a general in-hospital medical population.

After 6 years of implementation, we believe the practice of implementation of NEWS ${ }^{11}$ in the organizational context of IMC may provide to internists a practical answer rivalling the success of tools reported in other hospital settings (i.e. check lists in surgery or intensive care) to improve drastically safety and streamline processes of care. This in turn may provide guidance for health services researchers and decision makers in Italy and elsewhere on the optimal actions to take in the face of rising global challenges affecting 
acute medical wards.

Continued investment in safety and quality improvement within the medical ward deserve direct commitment and resources that have to be addressed towards what really works and may improve ward care deficiencies.

\section{References}

1. Pietrantonio F, Orlandini F, Moriconi L, La Regina M. Acute Complex Care Model: An organizational approach for the medical care of hospitalized acute complex patients. Eur J Intern Med 2015;26:759-65.

2. Nardi R, Mathieu G, La Regina M. Profilo biosociale del paziente ospedalizzato del XXI secolo. Quad Ital J Med 2018;6:4-8.

3. Chesi G, Boni F. Hospitals and organizational models based on the intensity of treatment: the internist's point of view. Ital J Med 2012;6:63-71.

4. Pannick S, Davis R, Ashrafian H, et al. Effects of interdisciplinary team care interventions on general medical wards: a systematic review. JAMA Intern Med 2015; 175:1288-98.

5. Pannick S, Beveridge I, Wachter RM, Sevdalis N. Improving the quality and safety of care on the medical ward: a review and synthesis of the evidence base. Eur J Intern Med 2014;25:874-87.

6. McQuillan P, Pilkington S, Allan A, et al. Confidential inquiry into quality of care before admission to intensive care. BMJ 1998;316:1853-8.

7. Seward E, Greig E, Preston S, et al. A confidential study of deaths after emergency medical admission: issues relating to quality of care. Clin Med Lond Engl 2003;3:425-34.

8. Torri E, Rigoni M, Dorigoni S, et al. A model based on intensity of medical care may improve outcomes for internal medicine patients in Italy. PLoS One 2019 [Epub ahead of print].

9. Ranhoff AH, Rozzini R, Sabatini T, et al. Subintensive care unit for the elderly: a new model of care for critically ill frail elderly medical patients. Intern Emerg Med 2006;1:197-203.

10. Recine U, Pastorelli R, Bruzzese V, et al. The change of hospital internal medicine: a study on patients admitted in internal medicine wards of 8 hospitals of the Lazio area. Ital J Med 2015;7:252-9.

11. Spagnolli W, Rigoni M, Torri E, et al. Application of the National Early Warning Score (NEWS) as a stratification tool on admission in an Italian acute medical ward: a perspective study. Int J Clin Pract 2017;71:3-4.

12. Royal College of Physicians. National Early Warning Score (NEWS) 2: standardising the assessment of acuteillness severity in NHS. Updated report of a working party. [Internet]. RCP London. 2017 [cited 2018 Mar 21]. Available from: https://www.rcplondon.ac.uk/projects/outputs/national-early-warning-score-news-2

13. Royal College of Physicians. National Early Warning Score (NEWS): standardising the assessment of acuteillness severity in NHS. Report of a working party. Royal College of Physicians; 2012.

14. Consiglio Direttivo FADOI Emilia Romagna. Medicina
Interna e organizzazione ospedaliera per intensità di cure: la posizione di FADOI Emilia Romagna. Ital J Med 2012;6:72-74.

15. Nardi R, Berti, F, Fabbri LM. Towards a sustainable and wise healthcare approach: potential contributions from hospital Internal Medicine Departments to reduce inappropriate medical spending. Ital J Med 2013;65-81.

16. Stefani I, Mazzone A. Come organizzare l'Area Medica per affrontare le nuove sfide della complessità clinica e assistenziale. Ital J Med 2014;2:54-9.

17. Vincent J-L, Rubenfeld GD. Does intermediate care improve patient outcomes or reduce costs? Crit Care Lond Engl 2015;19:89.

18. Alessandri M, Bartolomei C, Bernardini M, et al. Medicina Interna e nuova organizzazione ospedaliera: la proposta di FADOI Toscana. Ital J Med 2007;1:65-9.

19. Fernandes L, Duque S, Silvestre J, et al. Prognostic factors of patients admitted in a medical intermediate care unit: a prospective observational study. Int Arch Med [Internet] 2015;30:8.

20. Favaretti C, De Pieri P, Torri E, et al. An EFQM excellence model for integrated healthcare governance. Int $\mathrm{J}$ Health Care Qual Assur 2015;28:156-72.

21. Ham C, Kipping R, McLeod H. Redesigning work processes in health care: lessons from the National Health Service. Milbank Q 2003;81:415-39.

22. McGlynn EA, Asch SM, Adams J, et al. The quality of health care delivered to adults in the United States. N Engl J Med 2003;348:2635-45.

23. Lilford R, Mohammed MA, Spiegelhalter D, Thomson R. Use and misuse of process and outcome data in managing performance of acute medical care: avoiding institutional stigma. Lancet Lond Engl 2004;363:1147-54.

24. Bodenheimer T, Bojestig M, Henriks G. Making systemwide improvements in health care: lessons from Jönköping County, Sweden. Qual Manag Health Care 2007; 16:10-5.

25. Groene O, Mora N, Thompson A, et al. Is the maturity of hospitals' quality improvement systems associated with measures of quality and patient safety? BMC Health Serv Res 2011;11:344.

26. Needleman J, Buerhaus P, Pankratz VS, et al. Nurse staffing and inpatient hospital mortality. N Engl J Med 2011;364:1037-45.

27. Edbrooke DL, Minelli C, Mills GH, et al. Implications of ICU triage decisions on patient mortality: a cost-effectiveness analysis. Crit Care Lond Engl 2011;15:R56.

28. Smith MEB, Chiovaro JC, O’Neil M, et al. Early warning system scores: a systematic review [Internet]. Washington (DC): Department of Veterans Affairs; 2014 [cited 2015 Dec 28. (VA Evidence-based Synthesis Program Reports). Available from: http://www.ncbi.nlm. nih.gov/books/NBK 259026/

29. Plate JD, Peelen LM, Leenen LP, Hietbrink F. Validation of the VitalPAC Early Warning Score at the Intermediate Care Unit. World J Crit Care Med 2018;7:39-45.

30. Serafini F, Fantin G, Brugiolo R, et al. Outlier admissions of medical patients: prognostic implications of outlying patients. The Experience of the Hospital of Mestre. Ital J Med 2015;9:299-302. 